\title{
- Retracted -
}

\section{CORRIGENDUM}

\section{PRIMA-1Met induces mitochondrial apoptosis through activation of caspase- 2}

J Shen, H Vakifahmetoglu, H Stridh, B Zhivotovsky and KG Wiman

Oncogene (2016) 35, 6446; doi:10.1038/onc.2016.210; published online 4 July 2016

Correction to: Oncogene (2008) 27, 6571-6580; doi:10.1038/onc. 2008.249

Following the publication of this paper, the authors noted some errors in the figures presented.

The authors repeated the experiments presented in Figure $5 c$ (western blot to show cytochrome $c$ release upon PRIMA-1MET treatment) and Supplementary Figure S2 (western blot to show Bid cleavage upon PRIMA-1MET treatment) with small modifications. In Figure $5 c$, the pan-caspase inhibitor zVAD was used instead of the caspase-3 inhibitor zDEVD. In Supplementary Figure S2, an antibody that detects full-length Bid was used

C
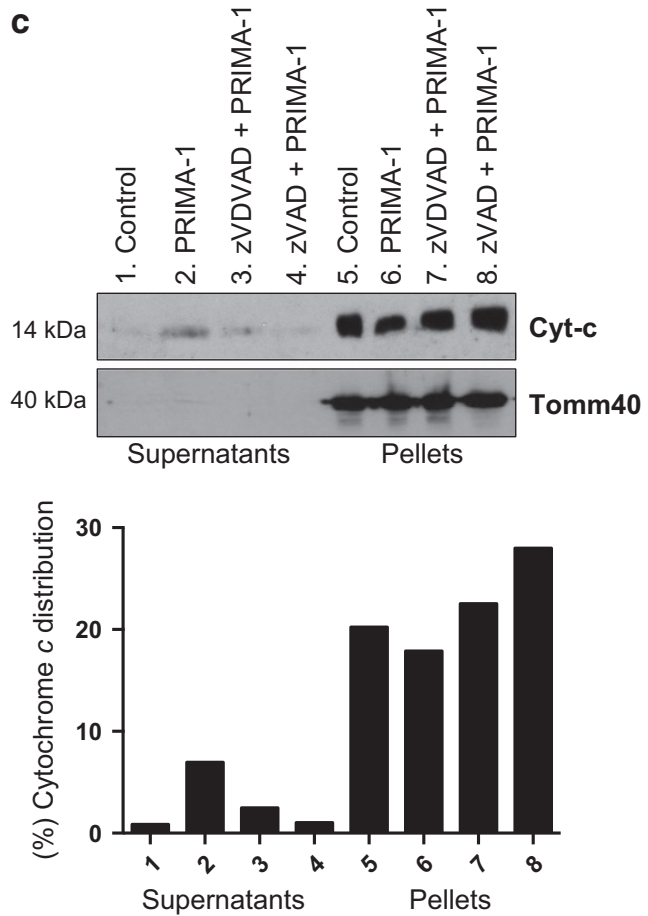

Figure 5. (c) Western blot analysis showing cytochrome $c$ distribution in supernatants and mitochondrial pellets (upper panel) of $\mathrm{H} 1299-\mathrm{His} 175$ cells after $16 \mathrm{~h}$ of treatment with $37.5 \mu \mathrm{M}$ PRIMA-1 ${ }^{\text {MET }}$. The mitochondrial protein Tomm40 was used as marker for the mitochondrial fraction. Quantification of protein levels is shown in the lower panel. The cytochrome $c$ antibody was from BD Biosciences, mAb clone 7H8.2C12, \#556433. The Tomm40 antibody was from Santa Cruz, Antibody H-300, sc-11414. rather than an antibody that detects both full-length and cleaved Bid.

In addition, a new version of Figure 6a (siRNA knockdown of caspase-2) was created using raw data.

The three revised figures are presented below along with figure legends.

All data are in agreement with previous findings described in the original article, and the main conclusion remains unchanged.

The authors apologise for any inconvenience caused.

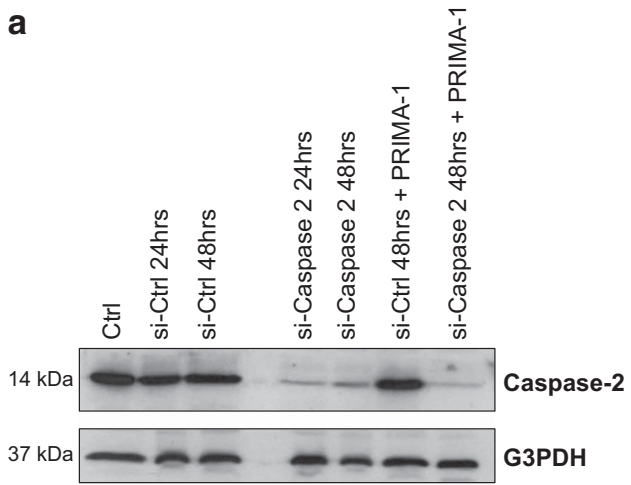

Figure 6. (a) Western blot analysis of caspase-2 expression. H1299His175 cells were treated with siRNA against caspase-2 for 24-48 h prior to treatment with $37.5 \mu \mathrm{M}$ PRIMA-1 ${ }^{\mathrm{MET}}$. A scrambled siRNA (siCtrl) was used as control. G3PDH was used as control for equal loading. The caspase-2 antibody was from BD Biosciences, mAb clone 35, \#611023.

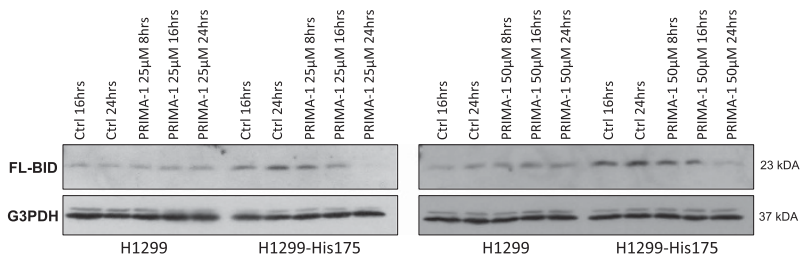

Figure S2. 